\title{
Least squares fitting of parametric surfaces to measured data
}

\author{
G. A. Watson * \\ (Received 7 August 2000)
}

\begin{abstract}
The problem is considered of fitting surfaces to measured data using the least squares norm, where it is assumed that a parameterization of the surface is available. Examples of practical applications include the product design and quality assurance of manufactured parts. There has been much recent algorithmic development based on conventional fitting ideas, mainly orthogonal distance regression. A different approach is taken here which explicitly takes account of the measurement process, and this is illustrated by some examples.
\end{abstract}

*Department of Mathematics, University of Dundee, Dundee DD1 4HN, Scotland.

${ }^{0}$ See http: //anziamj . austms .org. au/V42/CTAC99/AWat for this article and ancillary services, (c) Austral. Mathematical Soc. 2000. Published 27 Nov 2000. 


\section{Contents}

1 Introduction

C69

2 Fitting methods

C71

3 Some examples of surfaces

C78

3.1 An ellipsoid . . . . . . . . . . . . . . . . . . C79

3.2 An ellipsoidal cylinder . . . . . . . . . . . . . . . C80

3.3 A right circular cone . . . . . . . . . . . . . . . . C81

3.4 A torus ...................... C82

4 Some numerical examples $\quad$ C84

5 Conclusions $\quad$ C91

References

C92

\section{Introduction}

Let $m$ points $x_{i}, i=1, \ldots, m$ in $\mathbb{R}^{3}$ be obtained by sampling the surface of a manufactured part. Then the problem considered here is that of determining a model part (or equivalently a set of parameters defining the model part) which best fits those data. This kind of problem is a fundamental problem 
in metrology, where it is required to assess a manufactured part using data gathered by a coordinate measuring system.

Let a point on a surface in three dimensions be represented by

$$
x=f(\alpha, t),
$$

where $\alpha \in \mathbb{R}^{p}$ is a vector of $p$ parameters characterizing the nature of the surface and $t \in \mathbb{R}^{2}$ is a pair of scalar parameters fixing the position of the particular point $x$ on the surface. For example, for a sphere, $p=4$, with the 4 parameters giving the co-ordinates of the centre of the sphere and its radius; $t=(\theta, \phi)^{T}$ would be the usual trigonometric parameters of a point on the surface of the sphere.

In the following section, we consider some ways of fitting a model, characterized in this way, to given data. The criterion used is the least squares norm, although other criteria may be more appropriate on occasions. As well as describing conventional fitting methods, which take no account of how the data are obtained, we consider a method which exploits knowledge of the measurement process, when a coordinate measuring machine is used. This extends a technique recently discussed for curve fitting in [18]. Some examples of surfaces are given in Section 3, and some numerical results in Section 4. 


\section{Fitting methods}

For given $x_{i} \in \mathbb{R}^{3}, i=1, \ldots, m$, and $f$, let $\delta_{i} \in \mathbb{R}$ and $v_{i} \in \mathbb{R}^{3}$ satisfy

$$
x_{i}-f\left(\alpha, t_{i}\right)=\delta_{i} v_{i}, \quad v_{i}^{T} v_{i}=1, \quad i=1,2, \ldots, m .
$$

Then a standard approach to determining the correct model is to solve the optimization problem

$$
\text { minimize }\|\delta\|_{2}^{2} \quad \text { subject to (1). }
$$

This is a special case of orthogonal distance regression [3, 7, 14, 19], and corresponds to minimizing the least squares norm of the errors in the data. An alternative approach which corresponds to a residual minimization procedure is considered (in two dimensions) in [6, 17].

The constraints (1) can of course be eliminated, and so the problem is essentially the unconstrained minimization of

$$
\phi(\alpha, t)=\sum_{i=1}^{m}\left\|x_{i}-f\left(\alpha, t_{i}\right)\right\|_{2}^{2}
$$

with respect to $\alpha$ and $t=\left(t_{i}^{T}, \ldots, t_{m}^{T}\right)^{T}$. The variables of this problem partition naturally into two groups (and $t_{i}$ only occurs in the $i$ th term of the sum) and this has led to interest in methods which exploit the structure, based on separation of variables or decomposition. Let us assume that it is 
relatively easy to minimize each term of the sum in (2) with respect to $t_{i}$ (and therefore the sum with respect to $t$ ) for fixed $\alpha$, and for any $\alpha \in \mathbb{R}^{p}$, define the value function

$$
v(\alpha)=\min _{t} \phi(\alpha, t)
$$

Then the problem can be restated as

$$
\text { find } \alpha \text { to minimize } v(\alpha) \text {, }
$$

a problem in $\mathbb{R}^{p}$ only. An iteration of the method can therefore be stated:

$$
\text { find } t^{(k)}=\arg \min _{t} \phi\left(\alpha^{(k)}, t\right) \text {, update } \alpha^{(k)} \text { to } \alpha^{(k+1)} \text {. }
$$

When $f$ is linear in $\alpha$, updates can readily be obtained by a second minimization with $t^{(k)}$ fixed (see, for example, Späth [13]), but the method is then just the alternating algorithm and performance can be poor. Alternatively, whether $f$ is linear or nonlinear, a step of the Gauss-Newton or Levenberg-Marquardt method can be taken to give $\alpha^{(k+1)}$. In particular, the Gauss-Newton step $d$ will solve the problem

$$
\min _{d}\|\delta+J d\|_{2}^{2}
$$

where $J=\nabla_{\alpha} \delta$. Because $\nabla_{t} \delta=0$ at $t=t^{(k)}$, then this is the correct Gauss-Newton step in $\alpha$ taking account of the dependence of $\alpha$ on $t$. Of course $J$ is easily computed. The resulting iteration process (for both linear 
and nonlinear problems) seems effective: for some evidence, see $[15,16]$. A trust region variant is given in [8]. Note that the solution of the "foot point problem" for $t^{(k)}$ can, for surfaces, be nontrivial (see, for example, $[1,2,15]$ ).

The data points $x_{i}$ are often obtained using a coordinate measuring machine. This is a device in which a probe moving in a particular direction identifies a point on the part surface; the part is moved (for example rotated) with respect to the machine and this operation is repeated. Sometimes (although not always), it is possible to identify to good accuracy the probe directions for each data point, with respect to a particular frame of reference. Suppose that the $i$ th probe direction is known to be $v_{i}$, normalized so that $v_{i}^{T} v_{i}=1$. Then if the size of the probe head is ignored, the probe head will make contact with the manufactured part at the point $x_{i}$ and with the model part at the point given by $f\left(\alpha, t_{i}\right)$. Let $\delta_{i}$ denote the distance between these points; then

$$
x_{i}-f\left(\alpha, t_{i}\right)=\delta_{i} v_{i}, i=1,2, \ldots, m,
$$

and we require to minimize $\|\delta\|_{2}^{2}$ subject to these conditions. Comparison with (1) shows that these systems of equations are identical; however the problems are not, because now $v_{i}$ is fixed and no longer a parameter of the problem. Therefore it can no longer be eliminated as before. This fitting criterion is due to Hulting [9], who claims that the appeal of the approach lies not just in the use of the measurement design, but also in its compliance with traditional fixed-regressor assumptions (enabling standard inference theory to apply).

The advantage of dealing with an unconstrained problem seems to have 
been lost. However, this is not really the case because for each $i$ we can eliminate the 2 components of $t_{i}$ between the 3 scalar equations making up (1), to obtain a single equation for $\delta_{i}$. Then the real roots of that equation will give the points where the line defined by the point $x_{i}$ and the direction $v_{i}$ cuts the surface. Of course there may be no roots if the line through $x_{i}$ in the direction $v_{i}$ does not cut the model surface. However, if there is at least one real root, then we can identify uniquely the smallest value, and this we will refer to (without ambiguity) as $\delta_{i}$ : then $\delta_{i}$ and $t_{i}$ (through its dependence on $\delta_{i}$ ) can be considered as functions of $\alpha$. If all $\delta_{i}, i=1, \ldots, m$ exist, then $\|\delta\|_{2}$ measures the distance of the actual surface from the model one, and is a function of $\alpha$ only. We can in that case write

$$
F(\alpha)=\|\delta\|_{2}^{2}
$$

Notice that the calculation leading to this replaces the "foot point" calculation of the conventional approach, and can often be considerably simpler.

In addition to function values, derivatives of $\delta$ with respect to the components of $\alpha$ can usually be computed, and so derivative methods can be implemented. Dropping subscripts $i$, the relation connecting relevant quantities is

$$
x-\delta v=f(\alpha, t) .
$$

Differentiating through with respect to $\alpha$ gives

$$
-v \nabla_{\alpha} \delta=\nabla_{\alpha} f+\frac{\partial f}{\partial t_{1}} \nabla_{\alpha} t_{1}+\frac{\partial f}{\partial t_{2}} \nabla_{\alpha} t_{2},
$$


or

$$
\left[v: \frac{\partial f}{\partial t_{1}}: \frac{\partial f}{\partial t_{2}}\right]\left[\begin{array}{c}
\nabla_{\alpha} \delta \\
\nabla_{\alpha} t_{1} \\
\nabla_{\alpha} t_{2}
\end{array}\right]=-\nabla_{\alpha} f,
$$

a system of $3 p$ equations in $3 p$ unknowns. Thus

$$
\nabla_{\alpha} \delta=-e_{1}^{T}\left[v: \frac{\partial f}{\partial t_{1}}: \frac{\partial f}{\partial t_{2}}\right]^{-1} \nabla_{\alpha} f,
$$

where $e_{1}$ is the first co-ordinate vector, provided that the matrix is nonsingular. Consider now conditions for the existence of derivatives.

Theorem 1 Assume that the line through $x_{i}$ in the direction $v_{i}$ cuts the surface defined by $\alpha$, but does not lie in the tangent plane to the surface at the point defined by $t_{i}$, then $\nabla_{\alpha} \delta_{i}$ exists at this value of $\alpha$.

Proof: For convenience, the subscript $i$ will be dropped. Then the tangent plane to the surface defined by $\alpha$ at the point defined by $t$ is perpendicular to the vector product $\frac{\partial f}{\partial t_{1}} \times \frac{\partial f}{\partial t_{2}}$, so that if $v_{i}$ lies in this plane,

$$
v^{T}\left(\frac{\partial f}{\partial t_{1}} \times \frac{\partial f}{\partial t_{2}}\right)=0 .
$$

It follows using standard vector analysis that

$$
\operatorname{det}\left[v: \frac{\partial f}{\partial t_{1}}: \frac{\partial f}{\partial t_{2}}\right]=0
$$


The result follows.

A consequence of the above is that the Gauss-Newton method (or variants which control the step size) can be used to solve the problem. In particular, the Gauss-Newton step $d$ is obtained by solving

$$
\min _{d}\|\delta+G d\|_{2}^{2}
$$

where $G=\nabla_{\alpha} \delta$. Note that, as in (5), the correct Gauss-Newton step is being computed.

For models whose orientation is not aligned with the coordinate axes, then either the rotation parameters can be included in the model parameterization, or alternatively the data can be rotated. For planar curves, rotating the data is recommended [6], and this can be done by incorporating an additional parameter. Data rotation in space (about the origin) of a single point can be described by two parameters, corresponding to the multiplication of the data by a Householder matrix

$$
W(\rho, \tau)=I-w w^{T},
$$

where

$$
w=(\cos \rho \sin \tau, \sin \rho \sin \tau, \cos \tau)^{T} .
$$

However, for a set of data, this is not sufficient, and it seems necessary to multiply the data by the product of 3 Givens (elementary rotation) matrices, corresponding to a sequence of rotations $\rho, \sigma, \tau$ in the $\left(x_{2}, x_{3}\right),\left(x_{1}, x_{3}\right)$ and 
$\left(x_{1}, x_{2}\right)$ planes respectively. The best fit can then be obtained to the rotated data, and a reverse rotation implemented to obtain the correct model for the original data. Let $\beta=(\rho, \sigma, \tau)^{T}$, and let

$$
\begin{aligned}
& \bar{x}_{i}(\beta)=W(\beta) x_{i}, i=1, \ldots, m, \\
& \bar{v}_{i}(\beta)=W(\beta) v_{i}, i=1, \ldots, m,
\end{aligned}
$$

where $W$ is the product of the 3 matrices referred to above. Then $\bar{x}_{i}(\beta)$ and $\bar{v}_{i}(\beta)$ are just $x_{i}$ and $v_{i}$ respectively rotated about the origin by an amount defined by $\rho, \sigma$ and $\tau$. By analogy with (1),

$$
\bar{x}_{i}(\beta)-\delta_{i} \bar{v}_{i}(\beta)=f\left(\alpha, t_{i}\right), \quad i=1, \ldots, m,
$$

permitting $t_{i}$ and $\delta_{i}$ to be defined as functions of the shape parameters $\alpha$ and $\beta$. Partial derivatives of $\delta$ with respect to the components of $\beta$ are readily computed from (7). Differentiating with respect to $\beta_{j}$ and dropping the subscript $i$ gives

$$
\frac{\partial W}{\partial \beta_{j}} x-\frac{\partial \delta}{\partial \beta_{j}} \bar{v}-\delta \frac{\partial W}{\partial \beta_{j}} v=\nabla_{t} f(\alpha, t) \frac{\partial t}{\partial \beta_{j}}, \quad j=1,2,3 .
$$

Thus as before

$$
\frac{\partial \delta}{\partial \beta_{j}}=e_{1}^{T}\left[\bar{v}: \frac{\partial f}{\partial t_{1}}: \frac{\partial f}{\partial t_{2}}\right]^{-1} \frac{\partial W}{\partial \beta_{j}}(x-\delta v), j=1,2,3,
$$

provided the matrix is nonsingular. In addition

$$
\nabla_{\alpha} \delta=-e_{1}^{T}\left[\bar{v}: \frac{\partial f}{\partial t_{1}}: \frac{\partial f}{\partial t_{2}}\right]^{-1} \nabla_{\alpha} f
$$


Note that each matrix $\frac{\partial W}{\partial \beta_{j}}$ will be a product of three matrices, only one of which has elements differentiated. The problem in the larger set of variables can be solved by the Gauss-Newton method under the same conditions as before. Note that this modifies what is done in [18] to properly align the vectors $v_{i}$.

The above strategy, with or without rotation, may be used for any 3dimensional shapes which can be given in parametric form. It may in fact be used for more complicated shapes made up of combinations of simpler (parameterizable) shapes, in conjunction with a method for segmentation (where the data points are grouped into sets each belonging to a different surface). There are many techniques available for segmenting surfaces from three dimensional data, see for example, [10,4], and this is not considered further here.

Let us now consider a few examples of simple parameterizable surfaces to illustrate the approach.

\section{Some examples of surfaces}

It is frequently the case that $f$ can be written in the form

$$
f=A(t) \alpha+h(t),
$$


where $A \in R^{3 \times p}, h \in R^{3}$. This gives an explicit separation of the shape parameters $\alpha$ from the position parameters $t$, and displays a linear dependence on $\alpha$. Indeed sometimes it has an even simpler form when $h$ can be taken to be zero. Some examples are now given and it is shown how the components of $\delta$ may be calculated.

\subsection{An ellipsoid}

For the case of an ellipsoid whose axes are parallel to the coordinate axes,

$$
f=(a+p \cos \phi \sin \theta, b+q \sin \phi \sin \theta, c+r \cos \theta)^{T},
$$

so that $\alpha=(a, b, c, p, q, r)^{T} \in R^{6}$, and $t=(\theta, \phi)^{T}$. The fitting problem has constraints

$$
x_{i}-\delta_{i} v_{i}=A\left(t_{i}\right) \alpha, \quad i=1, \ldots, m,
$$

where

$$
A(t)=\left[\begin{array}{cccccc}
1 & 0 & 0 & \cos \phi \sin \theta & 0 & 0 \\
0 & 1 & 0 & 0 & \sin \phi \sin \theta & 0 \\
0 & 0 & 1 & 0 & 0 & \cos \theta
\end{array}\right] .
$$

Eliminating $t_{i}$ from (8) is readily achieved, so that $\delta_{i}$ is the smallest modulus value solving the quadratic equation

$$
\sum_{j=1}^{3} \frac{\left(\left(x_{i}\right)_{j}-\delta_{i}\left(v_{i}\right)_{j}-\alpha_{j}\right)^{2}}{\alpha_{3+j}^{2}}=1, \quad l=1, \ldots, m
$$


If there is no real solution, this means that the line through $x_{i}$ in the direction $v_{i}$ does not cut the ellipsoid, and it is then necessary to expand it. For each such value of $\delta_{i}$ obtained, the corresponding values of $t_{i}=\left(\theta_{i}, \phi_{i}\right)$ are readily calculated from (8).

\subsection{An ellipsoidal cylinder}

Assuming that an ellipsoidal shaped cylinder has axis the $x_{3}$ axis, then we can write

$$
f=(a+p \cos \theta, b+q \sin \theta, z)^{T} .
$$

so that $\alpha=(a, b, p, q)^{T} \in R^{4}$, and $t=(\theta, z)^{T}$. The shape parameters are the usual planar ones, and the position parameters correspond to the usual angular position, and the distance along the $x_{3}$ axis. The fitting problem has constraints

$$
x_{i}-\delta_{i} v_{i}=A\left(t_{i}\right) \alpha+\left(e_{2}^{T} t_{i}\right) e_{3}, \quad i=1, \ldots, m,
$$

where

$$
A(t)=\left[\begin{array}{cccc}
1 & 0 & \cos \theta & 0 \\
0 & 1 & 0 & \sin \theta \\
0 & 0 & 0 & 0
\end{array}\right] .
$$

Eliminating $t_{i}$ from (9) is readily achieved. Clearly, $\delta_{i}$ is then the smallest modulus value solving the quadratic equation

$$
\frac{\left(\left(x_{i}\right)_{1}-\delta_{i}\left(v_{i}\right)_{1}-a\right)^{2}}{p^{2}}+\frac{\left(\left(x_{i}\right)_{2}-\delta_{i}\left(v_{i}\right)_{2}-b\right)^{2}}{q^{2}}=1, \quad i=1, \ldots, m .
$$


If there is no real solution, this means that the line through $x_{i}$ in the direction $v_{i}$ does not cut the cylinder, and it is then necessary to increase the cylinder radius. This situation is perhaps most likely for the initial approximation, and provided the cylinder parameters are adjusted to avoid difficulties as identified by Theorem 1, should not occur again.

For each such value of $\delta_{i}$ obtained, the corresponding values of $t_{i}=\left(\theta_{i}, z\right)$ are readily calculated from (9).

\subsection{A right circular cone}

For simplicity, consider the special case of a right circular cone whose axis is parallel to the $x_{3}$ axis. Then we can write

$$
f=(a+s \cos \theta, b+s \sin \theta, c-d s)^{T},
$$

where $\alpha=(a, b, c, d) \in R^{4}$ and $t=(\theta, s)^{T}$. The values of $a, b, c$ give the coordinates of the apex of the cone, and $d$ is the cotangent of the angle the side of the cone makes with the $x_{3}$ axis. In the position vector $t, \theta$ is the angle which defines the position of the point on the the surface of the cone with respect to a slice of the cone through the point and parallel to the $x_{1}, x_{2}$ plane, and $s$ is the distance of the point from the axis. The fitting problem has constraints

$$
x_{i}-\delta_{i} v_{i}=A\left(t_{i}\right) \alpha+h\left(t_{i}\right), \quad i=1, \ldots, m,
$$


where

$$
A(t)=\left[\begin{array}{cccc}
1 & 0 & 0 & 0 \\
0 & 1 & 0 & 0 \\
0 & 0 & 1 & -s
\end{array}\right],
$$

and $h=s(\cos \theta, \sin \theta, 0)^{T}$. Here $\delta_{i}$ is the smallest modulus value solving the quadratic equation

$$
\left(\left(x_{i}\right)_{1}-\delta_{i}\left(v_{i}\right)_{1}-a\right)^{2}+\left(\left(x_{i}\right)_{2}-\delta_{i}\left(v_{i}\right)_{2}-b\right)^{2}=\left(\left(x_{i}\right)_{3}-\delta_{i}\left(v_{i}\right)_{3}-c\right)^{2} /\left(d^{2}\right) .
$$

From this value, corresponding values of $t_{i}=\left(\theta_{i}, s_{i}\right)^{T}$ are readily obtained from (11).

\subsection{A torus}

As a final example, we consider a slightly more complicated shape, a torus generated by rotating an ellipse about an axis. Let us assume that it is aligned so that its axis is parallel to the $x_{3}$ axis. Then we have

$$
f=(a+\cos \phi(m+r \cos \theta), b+\sin \phi(m+r \cos \theta), c+s \sin \theta)^{T},
$$

What is last component here??

where the centre of the torus is $(a, b, c)^{T}$, the semi-axes of the ellipse are $r$ and $s$, and the distance from the centre of the torus to the centre of the ellipse is $m$, assumed greater than $r$ and $s$. Then

$$
\alpha=(a, b, c, m, r, s)^{T}
$$


and $t=(\theta, \phi)^{T}$, the parameter $\phi$ fixing the plane $x_{2}-b=\tan \phi\left(x_{1}-a\right)$ of an elliptical slice of the torus, and the parameter $\theta$ fixing the position of the point on the surface of the torus in that plane. The fitting problem has constraints

$$
x_{i}-\delta_{i} v_{i}=A\left(t_{i}\right) \alpha, \quad i=1, \ldots, m,
$$

where

$$
A(t)=\left[\begin{array}{cccccc}
1 & 0 & 0 & \cos \phi & \cos \phi \cos \theta & 0 \\
0 & 1 & 0 & \sin \phi & \sin \phi \cos \theta & 0 \\
0 & 0 & 1 & 0 & 0 & \sin \theta
\end{array}\right],
$$

It is not difficult to eliminate $t_{i}$ from (12), although the outcome is a quartic in $\delta_{i}$, reflecting the fact that it is possible for a line through $x_{i}$ in the direction $v_{i}$ to cut the torus 4 times. Therefore the calculation of the smallest $\delta_{i}$ is not quite as straightforward as in the previous examples. However, once this is done, the corresponding values of $\theta_{i}$ and $\phi_{i}$ are readily obtained, and the Gauss-Newton method can be implemented as described previously.

It is readily seen how many other parametrically defined surfaces, such as paraboloids of revolution, hyperboloids, helicoids, more general conoids etc can be treated in exactly the same way. 


\begin{tabular}{|c|cccccccccccc|}
\hline$x_{1}$ & 2 & 1 & -1 & -2 & 1.0 & 0.5 & -0.5 & -0.5 & -1.5 & -1.0 & -0.5 & -0.5 \\
$x_{2}$ & 0 & 0 & 0 & -1.0 & 0 & 0.5 & -0.5 & -1.0 & 0.5 & 0.0 & -1.0 & 1.0 \\
$x_{3}$ & 0 & -0.8 & -1.0 & 0 & 0.0 & -1.0 & -1.0 & 0.3 & 0.0 & -1.0 & 0.0 & 0.0 \\
\hline$v_{1}$ & -1 & -1 & 1 & -1 & -1 & 1 & -1 & 1 & 3 & 1 & 1 & 1 \\
$v_{2}$ & 0 & 0 & 0 & 0 & -1 & 1 & -1 & 2 & 1 & 0 & 2 & -2 \\
$v_{3}$ & 0 & 1 & 1 & 0 & 0 & -2 & -2 & 0 & 0 & 1 & 0 & 0 \\
\hline
\end{tabular}

TABLE 1: Ellipsoid data and probe directions

\section{Some numerical examples}

Programmes written in MATLAB have been used to obtain numerical results for some of the surfaces described in the previous section. This is illustrated by a few examples.

Example 1: Consider fitting an ellipsoid (in normal orientation) to the data given in Table 1, where we also list appropriate directions $v_{i}$ (not necessarily normalized).

Starting with the approximation

$$
\alpha=(0,0,0,2,1,1)^{T},
$$

the performance of the method is shown in Table 2, stopping when $\left\|\nabla_{\alpha} F\right\|_{2}$ is less than $1.0 \mathrm{e}-4$. 


\begin{tabular}{|c|c|c|c|}
\hline$k$ & $F$ & $\left\|\nabla_{\alpha} F\right\|_{2}$ & $\gamma$ \\
\hline 1 & 0.2628 & 2.0769 & 1 \\
2 & 0.1094 & 0.1683 & 1 \\
3 & 0.1084 & 0.0058 & 1 \\
4 & 0.1083 & 0.0039 & 1 \\
5 & 0.1083 & 0.0004 & 1 \\
6 & 0.1083 & 0.0002 & 1 \\
7 & 0.1083 & $4.5 \mathrm{e}-5$ & \\
\hline
\end{tabular}

TABLE 2: Performance for ellipsoid and Table 1 data

The final parameter values obtained are

$$
\alpha=(0.0627,-0.0232,0.0595,1.9046,1.0612,1.2065)^{T} .
$$

Allowing the data to rotate, starting from this approximation (with initially zero rotations), gives the results shown in Table 3. The strategy for choosing $\gamma$ was to start with $\gamma=1$ and halve in the event of either a larger value of the objective function being obtained or no solution for some $\delta_{i}$. The benefits to be gained by allowing rotation are clear. The final parameter values are in this case

$$
\alpha=(0.0753,0.0319,0.1358,2.1093,0.9977,1.2454)^{T},
$$

with (in radians) $\rho=0.1059, \sigma=0.1317, \tau=0.1565$. Reasonable accuracy in terms of the objective function value can be obtained (particularly in the 


\begin{tabular}{|c|c|c|c|}
\hline$k$ & $F$ & $\left\|\nabla_{\alpha, \beta} F\right\|_{2}$ & $\gamma$ \\
\hline 1 & 0.1083 & 0.6644 & 1 \\
2 & 0.0424 & 0.3939 & 0.5 \\
3 & 0.0237 & 0.6357 & 1 \\
4 & 0.0180 & 0.0205 & 1 \\
5 & 0.0180 & 0.0120 & 1 \\
6 & 0.0180 & 0.0004 & 1 \\
7 & 0.0180 & $3.6 \mathrm{e}-5$ & \\
\hline
\end{tabular}

TABLE 3: Performance for rotated ellipsoid and Table 1 data

latter case) while the gradient is still relatively large, and it may be sensible to use a different criterion to stop the iteration.

Example 2: Consider fitting an ellipsoidal cylinder (with axis parallel to the $x_{3}$ axis) to the data $(m=15)$ shown in Table 4 . The vectors $v_{i}$ were chosen to be the vectors pointing from the corresponding data points to the $x_{3}$ axis, in the $x_{1}, x_{2}$ plane, that is

$$
\left(v_{i}\right) j=-\left(x_{i}\right)_{j}, j=1,2, \quad\left(v_{i}\right)_{3}=0, i=1, \ldots, m,
$$

correctly normalised.

The performance of the method starting from

$$
\alpha=(0,0,1,2)^{T}
$$




\begin{tabular}{|l|rrrrrrrr|}
\hline$x_{1}$ & 1.1949 & 1.1910 & 0.8096 & 0.2567 & -0.4115 & -0.4132 & -0.9547 & -0.7879 \\
$x_{2}$ & -0.1079 & 0.6293 & 1.5428 & 1.6519 & 1.7667 & 1.0866 & 0.2021 & -0.7269 \\
$x_{3}$ & 0.3858 & 0.7093 & 0.8159 & 0.3102 & 0.5210 & 0.0553 & 0.7238 & 0.4778 \\
\hline$x_{1}$ & -0.4251 & -0.1356 & 0.6315 & 0.8743 & 1.2314 & 0.9913 & 1.0420 & \\
$x_{2}$ & -1.7736 & -2.0980 & -2.2067 & -1.4398 & -0.5597 & 0.2415 & 0.9445 & \\
$x_{3}$ & 0.4465 & 0.8337 & 0.6812 & 0.9869 & 0.8562 & 0.4897 & 0.6793 & \\
\hline
\end{tabular}

TABLE 4: Ellipsoidal cylinder data

\begin{tabular}{|c|c|c|c|}
\hline$k$ & $F$ & $\left\|\nabla_{\alpha} F\right\|_{2}$ & $\gamma$ \\
\hline 1 & 0.9314 & 5.6606 & 1 \\
2 & 0.2676 & 0.3580 & 1 \\
3 & 0.2615 & 0.0080 & 1 \\
4 & 0.2615 & 0.0002 & 1 \\
5 & 0.2615 & $9.1 \mathrm{e}-6$ & - \\
\hline
\end{tabular}

TABle 5: Performance for ellipsoidal cylinder and Table 4 data 


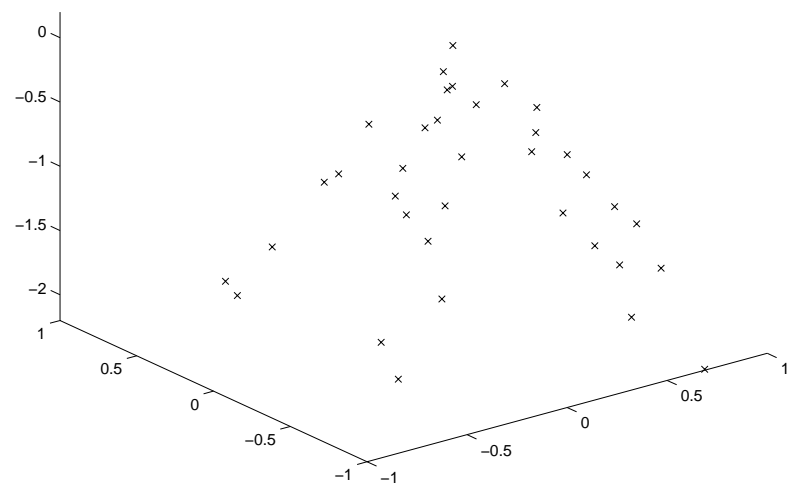

Figure 1: Cone data

is shown in Table 5. The final vector of parameters is

$$
\alpha=(0.1788,-0.2094,1.0186,2.0208)^{T} .
$$

Example 3: Consider fitting a right circular cone to the data points shown in Figure 1, which were generated by making random perturbations to 36 data points from the surface of the cone parametrized by $\alpha=(0,0,0,2)^{T}$. This means that the "ideal" cone (which is assumed to have axis parallel to the $x_{3}$ axis) has apex at the origin and the sloping side makes an angle $\cot ^{-1} 2$ with that axis. 


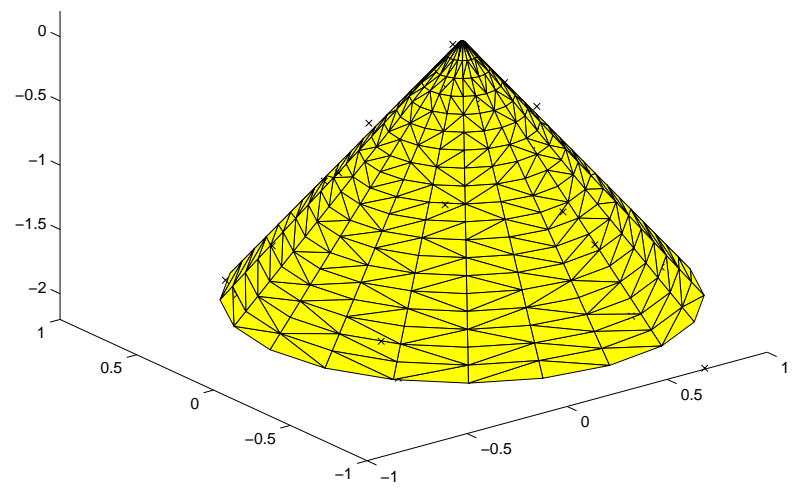

Figure 2: Model cone with data 


\begin{tabular}{|c|c|c|c|}
\hline$k$ & $F$ & $\left\|\nabla_{\alpha} F\right\|_{2}$ & $\gamma$ \\
\hline 1 & 0.8065 & 7.6181 & 1 \\
2 & 0.1887 & 1.3572 & 1 \\
3 & 0.1569 & 0.0689 & 1 \\
4 & 0.1568 & 0.0034 & 1 \\
5 & 0.1568 & 0.0001 & 1 \\
6 & 0.1568 & $5.6 \mathrm{e}-6$ & - \\
\hline
\end{tabular}

TABLE 6: Performance for right circular cone and Figure 1 data

The vectors $v_{i}$ were chosen to be the unit vectors pointing from the corresponding data points to the $x_{3}$ axis, in the $x_{1}, x_{2}$ plane, as in the previous example, except for that corresponding to the first data point (approximating the apex) which was $e_{3}$.

The performance of the method starting from

$$
\alpha=(0,0,0,2)^{T}
$$

is shown in Table 6. The final vector of parameters is

$$
\alpha=(0.1661,-0.0991,0.0928,2.0949)^{T} .
$$

Figure 2 shows the final model cone with the data. 


\section{Conclusions}

A method has been presented for fitting parametrically defined surfaces to data which makes explicit use of the probe directions when the data are obtained using a coordinate measuring machine. The method is based on the Gauss Newton method, but could be modified to include restrictions on the step size in a standard manner.

If the probe directions are not explicitly available (or even if they are), then traditional methods like orthogonal distance regression can be used. There may be an argument for using the present method even with estimated probe directions. For example, for symmetric shapes like spheres or ellipsoids, natural directions to take are those from the data to the centre, if a good estimate of that is available, as these would likely be aligned with the actual probe directions. That is effectively what is being done in Example 2 above. There are two other advantages: firstly, the potentially awkward foot point problem is avoided; secondly, the method readily generalises to other norms.

Finally, we point out that orthogonal distance regression techniques for offset surfaces is another possible alternative to the conventional approach: see for example $[5,11,12]$. 


\section{References}

[1] I.J. Anderson, M.G. Cox, A.B. Forbes, J.C. Mason, and D.A. Turner. An efficient and robust algorithm for the foot point problem. In M. Daehlen, T. Lyche, and L.L. Schumaker, editors, Mathematical Methods for Curves and Surfaces II, pages 9-16, Nashville, 1998, Vanderbilt University Press. C73

[2] I.J. Anderson, J.C. Mason, and D.A. Turner. An efficient algorithm for solving the surface foot point problem. In R. Cripps, editor, The Mathematics of Surfaces VIII, pages 89-103, London, 1998. Information Geometers. C73

[3] P.T. Boggs, R.H. Byrd, and R.B. Schnabel. A stable and efficient algorithm for nonlinear orthogonal distance regression. SIAM J. Sci. Stat. Comp., 8:1052-1078, 1987. C71

[4] R.M. Bolle and B.C. Vemuri. On three-dimensional surface reconstruction methods. IEEE Trans on Pattern Analysis and Machine Intelligence, 13:1-13, 1991. C78

[5] B.P. Butler, M.G. Cox, and A.B. Forbes. The reconstruction of workpiece surfaces from probe coordinate data. In R.B. Fisher, editor, Design and Application of Curves and Surfaces, Oxford, 1994. Oxford University Press. C91 
[6] W. Gander, G.H. Golub, and R. Strebel. Fitting of circles and ellipses: least square solution. BIT, 34:556-577, 1994. C71, C76

[7] H.-P. Helfrich and D. Zwick. A trust region method for implicit orthogonal distance regression. Numer. Alg., 5:535-545, 1993. C71

[8] H.-P. Helfrich and D. Zwick. A trust region algorithm for parametric curve and surface fitting. J. Comp. Appl. Math., 73:119-134, 1996. $\mathrm{C} 73$

[9] F.L. Hulting. Discussion contribution to the paper by M.M. Dowling, P.M. Griffin, K-L. Tsui, and C. Zhou. Statistical issues in geometric feature inspection using coordinate measuring machines. Technometrics, 39:18-20, 1997. C73

[10] P. Liong and J.S. Todhunter. Representation and recognition of surface shapes in range images: a differential geometry approach. Computer Vision, Graphics and Image Processing, 52:78-109, 1990. C78

[11] D. Sourlier. Three-dimensional Feature-Independent Bestfit in Coordinate Metrology. PhD Thesis, Swiss Federation Institute of Technology, Zurich, Switzerland, 1995. C91

[12] D. Sourlier and W. Gander. A new method and software-tool for the exact solution of complex dimensional measurement problems. In P. Ciarlini, M.G. Cox, F. Pavese, and D. Richter, editors, Advanced Mathematical Tools in Metrology II, Singapore, 1996. World Scientific Publishing Company. C91 
[13] H. Späth. Orthogonal least squares fitting by conic sections. In S. Van Huffel, editor, Recent Advances in Total Least Squares and Errors-in-Variables Techniques, pages 259-264, Philadelphia, 1997. SIAM. C72

[14] R. Strebel, D. Sourlier, and W. Gander. A comparison of orthogonal least squares fitting in coordinate metrology. In S. Van Huffel, editor, Recent Advances in Total Least Squares and Errors-in-Variables Techniques, pages 249-258, Philadelphia, 1997. SIAM. C71

[15] D.A. Turner. The Approximation of Cartesian Co-ordinate Data by Parametric Orthogonal Distance Regression. PhD Thesis, University of Huddersfield, 1999. C73, C73

[16] D.A. Turner, I.J. Anderson, J.C. Mason, M.G. Cox, and A.B. Forbes. An efficient separation-of-variables approach to parametric orthogonal distence regression. In P. Ciarlini, A.B. Forbes, F. Pavese, and D. Richter, editors, Advanced Mathematical and Computational Tools in Metrology IV, pages 246-255, Singapore, 2000. Series on Advances in Mathematics for Applied Sciences, Volume 53, World Scientific. C73

[17] J.M. Varah. Least squares data fitting with implicit functions. BIT, 36:842-854, 1996. C71

[18] G.A. Watson. Least squares fitting of circles and ellipses to measured data. BIT, 39:176-191, 1999. C70, C78 
[19] D.S. Zwick. Applications of orthogonal distance regression in metrology. In S. Van Huffel, editor, Recent Advances in Total Least Squares and Errors-in-Variables Techniques, Philadelphia, 1997. SIAM. C71 